\title{
A Method for Correcting The Error in Indicated Normal Acceleration Due to G- Sensor Location
}

\author{
Mi Yi \\ Shanghai Aircraft Design and Research Institute
}

\author{
Chen Mingtai \\ Shanghai Aircraft Design and Research Institute
}

\begin{abstract}
Particularly for structural purposes, it is important to obtain accurate measurements of the normal acceleration at the Center of Gravity of an aircraft. For most airliners and fighters, the g-sensors are located away from the Center of Gravity (C.G.). Theoretical mechanics shows that, depending on the distance between the g-sensor and the C.G., aircraft angular rates and accelerations will induce a difference between the actual normal acceleration at the C.G. and the normal acceleration indicated by the g-sensor. A method was devised to correct the normal acceleration indicated by the $g$ sensor so as to obtain the actual normal acceleration at the aircraft C.G. Based on the method presented in this paper, tests were conducted on the $B 737-800$ simulator and the corrections determined by the method were compared with the test data obtained from the simulator.
\end{abstract}

Keywords-Normal Acceleration, Correction, g-sensor, C.G., Aircraft

\section{INTRODUCTION}

Normally, in order to obtain the accurate normal acceleration of the aircraft, g-sensors should be located as close to the Center of Gravity (C.G.) as possible. However, for most airliners and fighters, g-sensors are usually located in the Electrical/Electronic cabinet as components of the Attitude Heading Reference System (AHRS) or the Inertial Navigation System (INS). Furthermore, the C.G. position of the aircraft changes in flight due to fuel consumption. It is important to determine the correction of the acceleration indicated by a g-sensor located away from the C.G. of the aircraft. Compared with the accelerations along $\mathrm{X}$ and $\mathrm{Y}$ axes, the normal acceleration is more important due to its effect on structural design.

\section{DEFINITIONS}

A. Body Axes

Figure. 1 shows the body axis notation and the velocities along and about each of the axes.

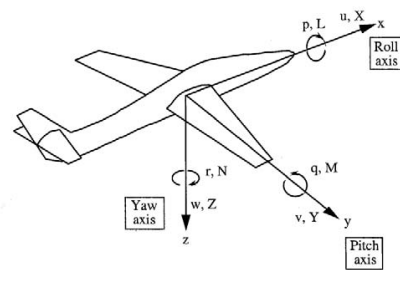

Figure 1. Body Axis System

$\mathrm{X}$ is along the longitudinal axis, $\mathrm{Y}$ is along the lateral axis and $\mathrm{Z}$ is along the normal axis. The motion of the aircraft described by the body axes system consists of three linear velocities $(\vec{u}, \vec{v}$ and $\vec{w})$ and three angular velocities ( $\vec{p}, \vec{q}$ and $\vec{r}$ ). The linear velocity $\vec{u}$, is defined as the component of the resultant linear velocity $\overrightarrow{V_{T}}$, which lies in the $X$ direction. Similarly, the rolling velocity $p$ is defined as the component of the total angular velocity $\omega$, which is about the $X$ axis. Positive directions are: $X$, forward along the fuselage; $Y$, out the right wing; $Z$, down through the bottom of the aircraft [1].

B. Acceleration

Acceleration is a vector quantity, and its unit is traditionally referred to as g (load factor), because it indicates the apparent acceleration of gravity sensed on board. Normal acceleration $(\mathrm{Nz})$ is the component of the linear acceleration of an aircraft along the $\mathrm{Z}$ axis. A normal acceleration of one, or $1 \mathrm{~g}$, represents conditions in straight and level flight, where the lift is equal to the weight.

Assume that the acceleration obtained by a g-sensor located away from the C.G. position is $\overline{a_{g-s e n s o r}}$ which contains three components along three axes:

$$
\overrightarrow{a_{g-\text { sensor }}}=a_{g-\text { sensor. } x} \vec{i}+a_{g-\text { sensor. } y} \vec{j}+a_{g-\text { sensor.z }} \vec{k}
$$

Assume that the acceleration at C.G. position is $\overline{a_{C . G}}$ :

$\overline{a_{C . G .}}=a_{C . G . x} \vec{i}+a_{C . G . y} \vec{j}+a_{C . G . z} \vec{k}$

Define that the correction of acceleration is $\overline{\Delta a}$ :

$$
\overrightarrow{\Delta a}=\Delta a_{x} \vec{i}+\Delta a_{y} \vec{j}+\Delta a_{z} \vec{k}
$$

The acceleration at the position of the $g$-sensor is equal to the sum of the acceleration at C.G. position and the correction caused by the g-sensor location away from the C.G.. As a component of the linear acceleration of the aircraft, normal acceleration is:

$$
a_{g-\text { sensor. } z}=a_{C . G . z}+\Delta a_{z}
$$

\section{REDUCTION}

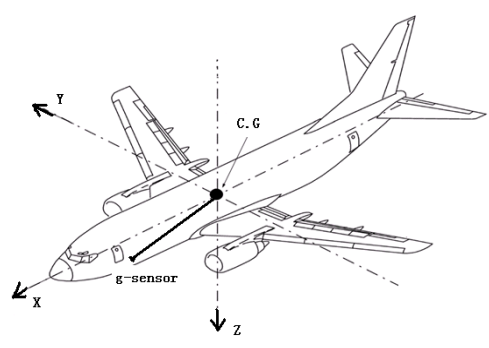

Figure 2. Location of g-sensor 
Assume that the aircraft is at rest and level. The location of the g-sensor is shown in Figure.2.

The C.G. position is $\left(x_{0}, y_{0}, z_{0}\right)$. Here, the C.G. is the origin of the body axes, so,

$$
\left(x_{0}, y_{0}, z_{0}\right)=(0,0,0)
$$

Assume that the g-sensor location is $\left(x_{g}, y_{g}, z_{g}\right)$, so the distance between the C.G. and the g-sensor is $\vec{R}:\left(R_{x}, R_{y}, R_{z}\right)$.

$$
\begin{aligned}
& R_{x}=x_{g}-x_{0}=x_{g} \\
& R_{y}=y_{g}-y_{0}=y_{g} \\
& R_{z}=z_{g}-z_{0}=z_{g}
\end{aligned}
$$

The acceleration of the g-sensor consists of the acceleration at the C.G. and the correction caused by the gsensor location away from the C.G. Here, only the correction was considered in following reduction.

\section{A. Linear Velocity of g-sensor}

Assume that the airplane has an instantaneous angular velocity $\bar{\omega}(p, q, r)$. The linear velocity [2] of g-sensor due to the arm and rotation around C.G. is:

$$
\begin{aligned}
& \overrightarrow{\Delta V}=\vec{\omega} \times \vec{R}=\left|\begin{array}{ccc}
\vec{i} & \vec{j} & \vec{k} \\
p & q & r \\
R_{x} & R_{y} & R_{z}
\end{array}\right| \\
& =\left(q R_{z}-r R_{y}\right) \vec{i}+\left(r R_{x}-p R_{z}\right) \vec{j}+\left(p R_{y}-q R_{x}\right) \vec{k}
\end{aligned}
$$

The three components of linear velocity are:

$$
\begin{aligned}
& \Delta u=q R_{z}-r R_{y} \\
& \Delta v=r R_{x}-p R_{z} \\
& \Delta w=p R_{y}-q R_{x}
\end{aligned}
$$

B. Acceleration Caused by the Location of the g-sensor

The resulting linear acceleration due to the location of the g-sensor is:

$$
\begin{aligned}
& \overrightarrow{\Delta a}=\frac{d \overrightarrow{\Delta V}}{d t}=\frac{d(\vec{\omega} \times \vec{R})}{d t} \\
& =\frac{d \vec{\omega}}{d t} \times \vec{R}+\vec{\omega} \times \frac{d \vec{R}}{d t}
\end{aligned}
$$

The first term is tangential acceleration and the second term is radial acceleration. Firstly, the angular velocity of the aircraft is $\bar{\omega}(p, q, r)$, so the differential of angular velocity can be written as:

$$
\frac{d \vec{\omega}}{d t}=(\dot{p}, \dot{q}, \dot{r})
$$

Then, the tangential acceleration is:

$$
\begin{aligned}
& \frac{d \vec{\omega}}{d t} \times \vec{R}=\left|\begin{array}{ccc}
\vec{i} & \vec{j} & \vec{k} \\
\dot{p} & \dot{q} & \dot{r} \\
R_{x} & R_{y} & R_{z}
\end{array}\right| \\
& =\left(\dot{q} R_{z}-\dot{r} R_{y}\right) \vec{i}+\left(\dot{r} R_{x}-\dot{p} R_{z}\right) \vec{j}+\left(\dot{p} R_{y}-\dot{q} R_{x}\right) \vec{k}
\end{aligned}
$$

Secondly, the radial acceleration is:

$$
\begin{aligned}
& \vec{\omega} \times \frac{d \vec{R}}{d t}=\vec{\omega} \times \overrightarrow{\Delta V} \\
& =\left|\begin{array}{ccc}
\vec{i} & \vec{j} & \vec{k} \\
p & q & r \\
\Delta u & \Delta v & \Delta w
\end{array}\right| \\
& =(q \cdot \Delta w-r \cdot \Delta v) \vec{i} \\
& +(r \cdot \Delta u-p \cdot \Delta w) \vec{j} \\
& +(p \cdot \Delta v-q \cdot \Delta u) \vec{k}
\end{aligned}
$$

The resultant acceleration is the sum of tangential acceleration and radial acceleration.

$$
\begin{aligned}
\overline{\Delta a} & =\frac{d \overrightarrow{\Delta V}}{d t}=\frac{d(\vec{\omega} \times \vec{R})}{d t}=\frac{d \vec{\omega}}{d t} \times \vec{R}+\vec{\omega} \times \frac{d \vec{R}}{d t} \\
& =\left[\left(\dot{q} R_{z}-\dot{r} R_{y}\right)+(q \cdot \Delta w-r \cdot \Delta v)\right] \vec{i} \\
& +\left[\left(\dot{r} R_{x}-\dot{p} R_{z}\right)+(r \cdot \Delta u-p \cdot \Delta w)\right] \vec{j} \\
& +\left[\left(\dot{p} R_{y}-\dot{q} R_{x}\right)+(p \cdot \Delta v-q \cdot \Delta u)\right] \vec{k}
\end{aligned}
$$

The resultant normal acceleration is:

$$
\overrightarrow{\Delta a_{z}}=\left(\dot{p} R_{y}-\dot{q} R_{x}\right) \vec{k}+(p \cdot \Delta v-q \cdot \Delta u) \vec{k}
$$

According to equation( $(8)$ :

$$
\begin{aligned}
& \Delta a_{z}=\left(\dot{p} R_{y}-\dot{q} R_{x}\right) \\
& +\left[p\left(r R_{x}-p R_{z}\right)-q\left(q R_{z}-r R_{y}\right)\right]
\end{aligned}
$$

Therefore, the correction caused by the location is:

$$
\begin{aligned}
& \Delta a_{z}=(p r-\dot{q}) R_{x} \\
& +(\dot{p}+q r) R_{y}-\left(p^{2}+q^{2}\right) R_{z}
\end{aligned}
$$

$\Delta a_{z}$ is the acceleration increment caused by the $\mathrm{g}$ sensor location away from the C.G. of the aircraft and it must be subtracted from the normal acceleration obtained from the g-sensor to obtain the normal acceleration at the C.G..

\section{INFLUENCING FACTORS ANALYSIS}

Based on the model presented above, the correction is determined by the distance between the g-sensor and the C.G. and the angular acceleration and angular velocity of the aircraft. Tests were conducted on the B737-800 simulator which gave the normal acceleration, pitch rate, yaw rate and roll rate of the aircraft. For the purpose of verifying the model, the aircraft was programmed to perform an unusual manoeuver. In this manoeuver, the yaw rate and roll rate were as large as the pitch rate [3]. The pitch rate, yaw rate, roll rate and normal acceleration at C.G. position are shown in Figure.3.

\section{A. Distance Effect}

In order to verify the effect of the distance between the g-sensor location and the C.G. position, assume that there are two g-sensors located at 10 meters and 15 meters along the $\mathrm{X}$ axis, respectively. Based on the pitch rate, yaw rate, roll rate and normal acceleration presented above, the correction to the normal acceleration indicated by the two gsensors was calculated and is shown in Figure.4. 
Observation of Figure. 4 shows that, when the distance between the g-sensor and the C.G. was 10meters, the maximum correction of normal acceleration was $0.32 \mathrm{~g}$, and the minimum correction was $-0.36 \mathrm{~g}$. When the distance was changed to 15 meters, the maximum correction of normal acceleration was $0.66 \mathrm{~g}$, and the minimum correction was $0.67 \mathrm{~g}$. Without changing angular velocity, the correction of normal acceleration changed substantially due to the distance variation. It can be concluded that the correction of normal acceleration increases with the distance between the g-sensor and the C.G. while keeping other variables constant.

B. Angular Velocity and Angular Acceleration Effects

The time history in Figure.5 shows the variation of the normal acceleration correction with pitch rate and pitch acceleration.

As shown in Figure.5, the correction of normal acceleration has a relationship with angular velocity and angular acceleration. When the angular acceleration is small (which means the angular velocity changes slowly), the correction of normal acceleration was nearly zero $g$ which can be neglected in practical flight. However, when the angular acceleration is large (which means the angular velocity changes rapidly), the correction of normal acceleration was $0.36 \mathrm{~g}$ which cannot be neglected in practical flight. It can be concluded that when the angular velocity changes more rapidly (the angular acceleration is large), the correction of normal acceleration will be substantial while keeping the distance variables constant.

\section{FURTHER RESEARCH}

According to the model, there are eight parameters required to determine the correction of normal acceleration: $R_{x}, R_{y}, R_{z}, p, q, r, \dot{p}, \dot{q}$. However, based on the specific characteristics of large aircraft such as airliners, the model can be simplified. Here, assume that the g-sensor is located close to the X-Y plane, then,

$$
\begin{aligned}
& R_{x} \neq 0 \\
& R_{y} \approx 0 \\
& R_{z} \neq 0
\end{aligned}
$$

According to equation (16):

$$
a_{z}=(p r-\dot{q}) R_{x}+(\dot{p}+q r) R_{y}-\left(p^{2}+q^{2}\right) R_{z}
$$

So, in this case, $a_{z}$ can be simplified as:

$$
a_{z}=(p r-\dot{q}) R_{x}-\left(p^{2}+q^{2}\right) R_{z}
$$

Furthermore, for an airliner, compared with the pitch rate and pitch acceleration, the roll and yaw rate are small in normal flight, so they can be neglected, therefore:

$$
\begin{aligned}
p r & \approx 0 \\
p^{2} & \approx 0
\end{aligned}
$$

Finally, equation (16) can be simplified as:

$$
a_{z}=-\dot{q} R_{x}-q^{2} R_{z}
$$

Consequently, just three variables are needed to make the correction, as follows: pitch rate, $q$, and the distance between the g-sensor and the C.G. $R_{x}$ and $R_{z}$ (the pitch acceleration $\dot{q}$ can be calculated from $q$ ).

\section{SUMMARY}

It has been shown that the correction of the normal acceleration as indicated by an "away from the C.G." gsensor is determined by the distance between the g-sensor and the C.G. position, angular velocity and angular acceleration. The correction model given in this paper was not tested on an actual aircraft due to time constraints and the availability of test aircraft. Validating tests should be conducted using test aircraft.

\section{REFERENCES}

[1] Dr. Robert C. Nelson. Flight Stability and Automatic Control [M]. McGraw-Hill Book Company, 1989.

[2] JIANG Yong-zheng, Simulator Motion System and Software Dissect, Journal of UEST of China [J], 2002,8, PP374-378

[3] YOU Jun-sheng; YOU Yong; XU Xu-yao; MA Yue-xin. Research for Modeling and Simulation of Pilot Three-shaft Acceleration, Journal of System Simulation [J], 2006.8, PP28-30. 

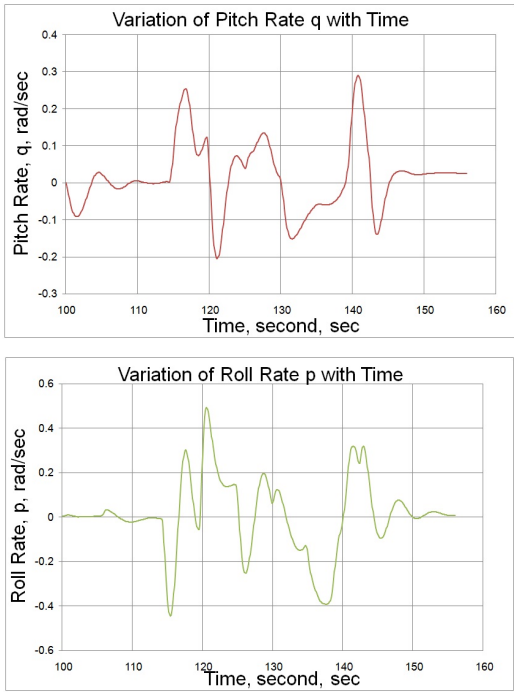
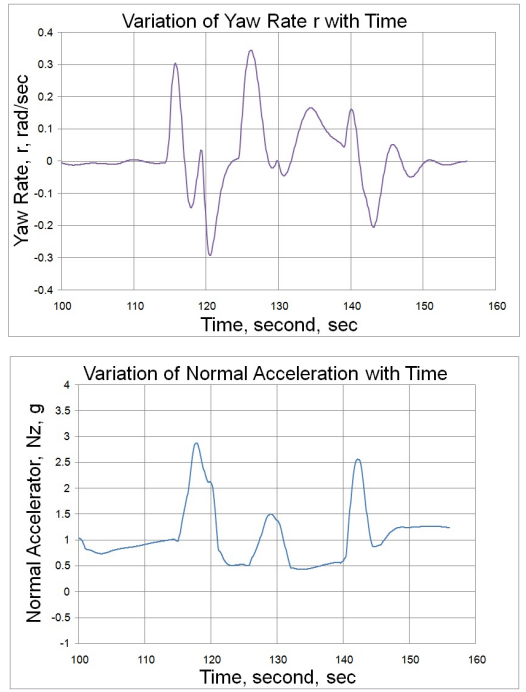

Figure 3. Variation of Velocity and Normal Acceleration with Time
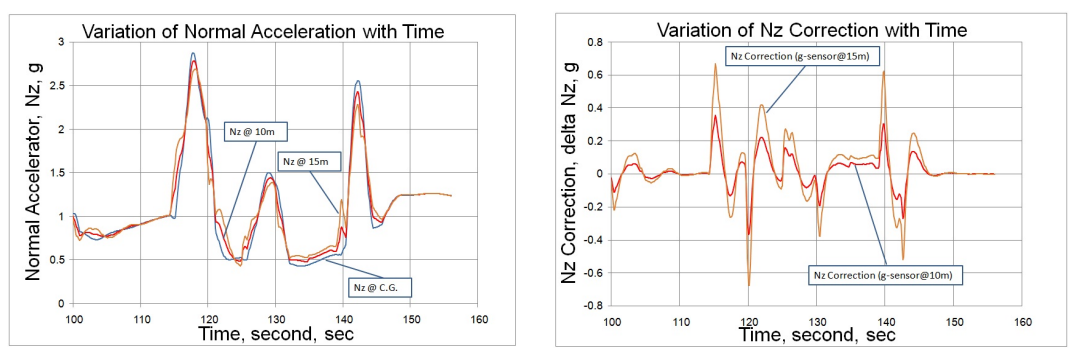

Figure 4. Nz Correction with Time
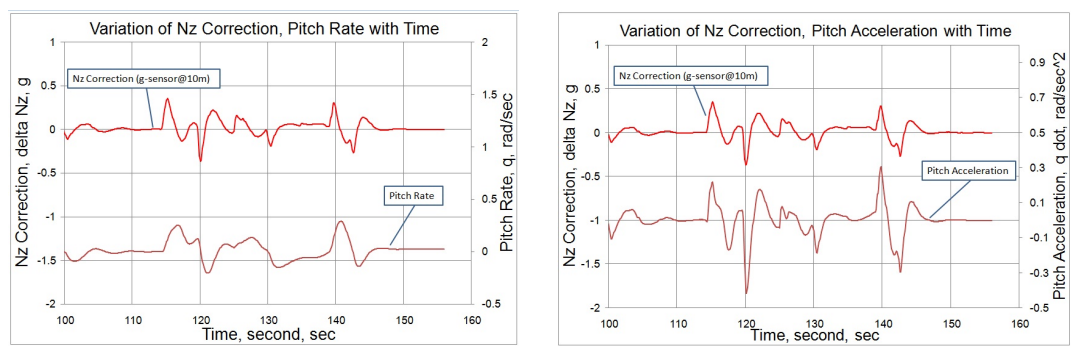

Figure 5. Nz Correction due to Pitch Rate and Pitch Acceleration 